\title{
Técnica de Delorme como Opção para o Tratamento da Procidência Retal Recidivada: Relato de Caso
}

\author{
Delorme's Technique as an Option for the Treatment of the Rectal \\ Prolapse Recurrence: Case Report
}

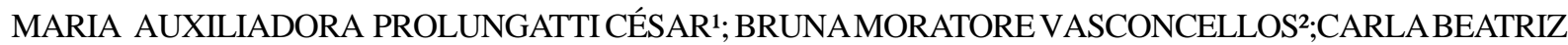 \\ CARNEIRODA CUNHA SOARES ${ }^{3}$;CAROLINA CALAFIORIDECAMPOS ${ }^{4}$;CAMILA SALERA ${ }^{5}$;DANIELA \\ BORTMAN $^{6}$;GABRIEL CONTREIRASANSONI ${ }^{7}$ JÚLIAMOTALEITE ${ }^{8}$;LUCIANA VENDRAMEL TENÓRIO \\ GOMES $^{9}$;NINAROSA KONICHI DA SILVA ${ }^{10}$;RAFAELACRISTINACOELHO MUNIZ ${ }^{11}$;RENATAMARTINS CORADINI ${ }^{12}$
}

\begin{abstract}
${ }^{1}$ Mestre e Doutora em Medicina pela Faculdade de Ciências Médicas da Santa Casa de São Paulo. Professora Assistente
Doutor do departamento de Medicina da Universidade de Taubaté; ${ }^{2}$ Aluna do Quarto ano do Departamento de

Medicina da Universidade de Taubaté; ${ }^{3}$ Aluna do Quarto ano do Departamento de Medicina da Universidade de

Taubaté $;{ }^{4}$ Aluna do Quarto ano do Departamento de Medicina da Universidade de Taubaté; ${ }^{5}$ Aluna do Quarto ano do

Departamento de Medicina da Universidade de Taubaté; ${ }^{6}$ Aluno do Quarto ano do Departamento de Medicina da

Universidade de Taubaté $;^{7}$ Aluna do Quarto ano do Departamento de Medicina da Universidade de Taubaté $;^{8}$ Aluna do

Quarto ano do Departamento de Medicina da Universidade de Taubaté; ${ }^{9}$ Aluna do Quarto ano do Departamento de

Medicina da Universidade de Taubaté ${ }^{10}$ Aluna do Quarto ano do Departamento de Medicina da Universidade de

Taubaté, ${ }^{11}$ Aluna do Quarto ano do Departamento de Medicina da Universidade de Taubaté, ${ }^{12}$ Aluna do Quarto ano do

Departamento de Medicina da Universidade de Taubaté.
\end{abstract}

CÉSAR MAP; VASCONCELLOS BM; SOARES CBCC; CAMPOS CC; SALERA C; BORTMAN D; SANSONI GC; LEITE JM; GOMES LVT; SILVA NRKS; MUNIZ RCC; CORADINI RM. Técnica de Delorme como Opção para o Tratamento da Procidência Retal Recidivada: Relato de Caso. Rev bras Coloproct, 2010;30(1): 083-086.

RESUMO: O prolapso retal é uma protrusão de todas as camadas do reto no sentido anal caracterizando um aspecto clínico de um tumor anal, inicialmente ele resulta de um esforço intenso com uma redução espontânea e posteriormente ele resulta de um esforço menor com dificuldade na redução. A incidência é maior em mulheres. O diagnóstico é essencialmente clínico e o tratamento é cirúrgico. Relato de caso: NM, 65 anos, diabético e hipertenso, submetido a sacropromontofixação associada a técnica de Moscowicz com recidiva após um mês, então a técnica de Delorme foi utilizada com sucesso. Discussão: os procedimentos abdominais são indicados para pacientes com menor risco cirúrgico devido a grande complexidade e menor taxa de recidiva. Dentre os procedimentos perineais, a técnica de Delorme possui uma aplicação simples, contudo com uma alta taxa de recidiva. A técnica de Delorme possui uma menor taxa de mortalidade e foi a melhor conduta para este paciente.

Descritores: Prolapso retal, cirurgia coloretal, procedimento operatório cirúrgico.

\section{INTRODUÇÃO}

O prolapso retal tem sido documentado desde as civilizações egípcia e grega e ainda existem muitas controvérsias sobre o tratamento ${ }^{1,2,3,4}$. A procidência ou prolapso total é uma protrusão de todas as camadas do reto através do orifício anal, formando uma verda- deira hérnia por deslocamento através do diafragma pélvico5. Ocorre com maior freqüência em pacientes idosos do sexo feminino, nos homens ocorre principalmente entre os 20-40 anos.

Dentre as principais podemos encontrar ${ }^{6}$ :

- Profundidade anormal do Fundo de Saco de Douglas;

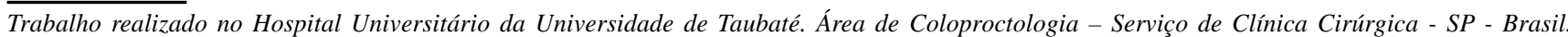

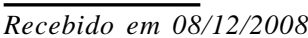

Aceito para publicação em 07/04/2009 
- Atonia ou frouxidão do diafragma pélvico e do canal anal;

- Falta de fixação do reto aos tecidos adjacentes.

O quadro clínico é de tumoração anal inicialmente aos grandes esforços com redução espontânea e posteriormente aos pequenos esforços com difícil redução. Ao exame físico observa-se protusão do reto, com uma massa durante e após a evacuação. A mucosa do seguimento exteriorizado apresenta-se congesta, recoberta de muco, às vezes ulcerada, e tem pregas caracteristicamente concêntricas, não evidentes quando há muito edema. Em relação aos exames complementares a endoscopia digestiva alta é importante apenas para descartar patologias concomitantes ${ }^{7}$. A defecografia pode auxiliar no diagnóstico de intussuscepção oculta e a manometria confirma a hipotonia esfincteriana presente nesses casos ${ }^{8}$. O tratamento é cirúrgico e a abordagem pode ser abdominal ou perineal.

Historicamente, a correção da procidência retal tem envolvido desde técnicas perineais simples, como a operação de Thiersch, até técnicas cirúrgicas mais complexas, dentre elas a retopexia, que é feita por um acesso abdominal, e a Delorme, que é uma das técnicas de acesso perineal.

\section{OBJETIVO}

Relato de caso de paciente com procidência retal tratado com a técnica de Delorme.

\section{CASO CLÍNICO}

NM, 65 anos, diabético e hipertenso, com história de procidência retal há cinco anos e que se submeteu à sacropromontofixação associado à Moscowicz com recidiva após um mês.

No exame proctológico apresentava orifício anal entreaberto, mucosa anal exteriorizada e presença de restos fecais na margem anal. Na inspeção dinâmica apresentava exteriorização de todas as camadas do reto e ao toque retal esfíncter hipotônico, perveo para mais que uma polpa digital (Figura 1).

A colonoscopia foi normal e a manometria demostrou canal anal curto e hipotonia esfincteriana interna e externa.

Realizado preparo intestinal retrógrado e a cirurgia foi realizada com anestesia raquidural.
A técnica cirúrgica se baseou nos seguintes passos:

1-Paciente em posição de litotomia sob anestesia raqui

2-Exposição do prolapso com Allis

3- Infiltração da mucosa retal com SF e solução de adrenalina na proporção de

1: 300.000 (Figura 2)

4-Incisão circular da mucosa e submucosa retal $1 \mathrm{~cm}$ acima da linha pectínea com lâmina 15 .

5- Descolamento mucoso até atingir o ápice do prolapso retal. Hemostasia realizada com eletrocoagulação. (Figura 3)

6- Emprego dos fios absorvíveis para suturas da camada muscular do reto, realizando dois pontos em cada quadrante, imbricando e suturando a camada muscular do reto.

7- Secção e excisão da mucosa excedente.

8- Sutura contínua com fios absorvíveis (categut cromado) da camada mucosa retal junto a canal anal centímetro acima da linha pectínea.

9- Curativo oclusivo.

No pós-operatório o paciente não apresentou queixas ou intercorrências, sem apresentar recidiva da procidência retal após seis meses de acompanhamento ambulatorial.

\section{DISCUSSÃO}

O tratamento da procidência retal é cirúrgico ${ }^{6}$. A escolha do procedimento a ser utilizado é discutível, já que nenhuma técnica é completamente eficaz. Os procedimentos abdominais, em função de sua maior

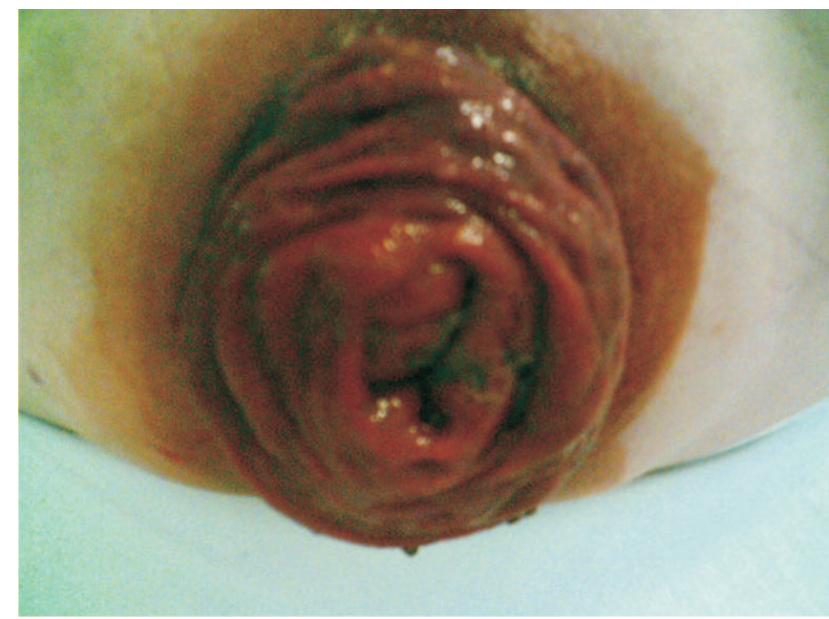

Figura 1 - Procidência retal. 


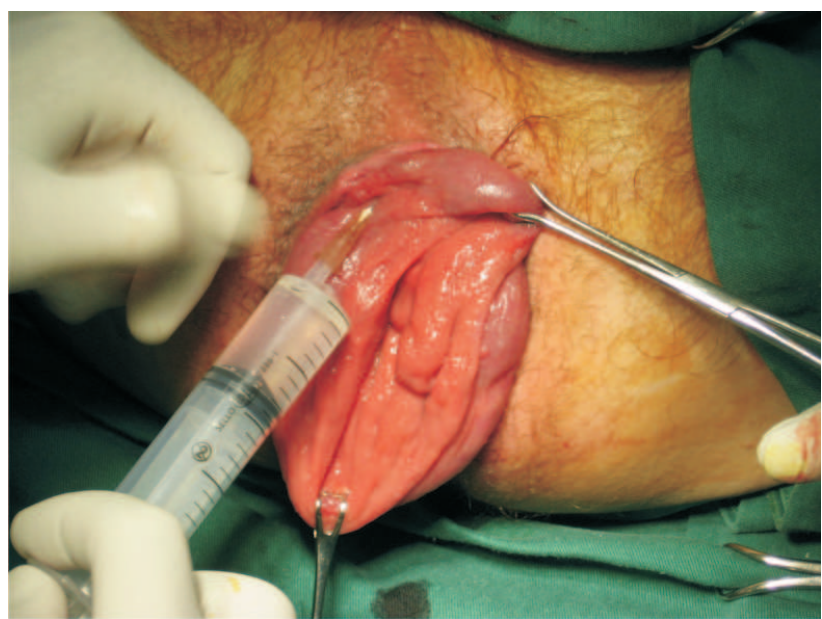

Figura 2 - Início da cirurgia.

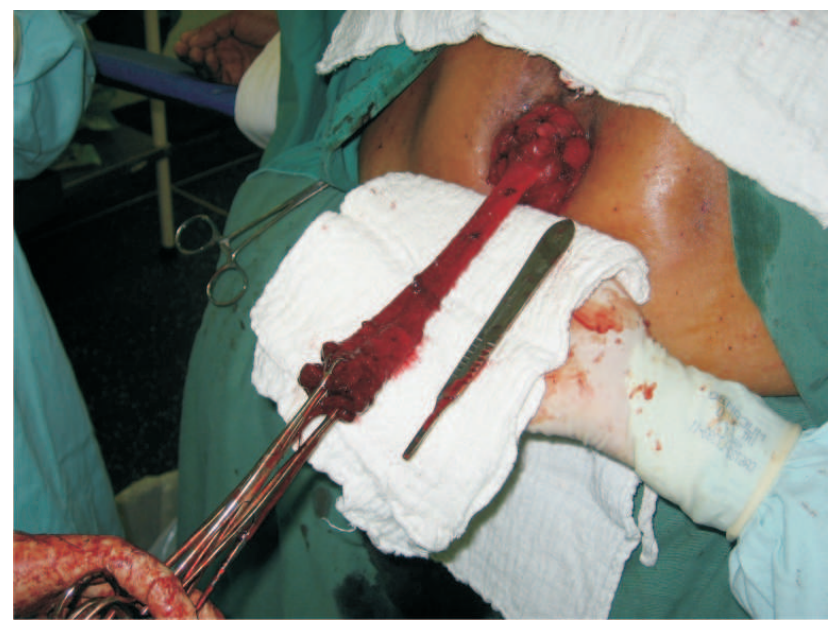

Figura3 - Aspecto final da dissecção.

complexidade, são reservados para pacientes com um risco cirúrgico menor. As correções perineais, de execução mais simples e sem necessidade de anestesia geral, são indicadas para pacientes de maior risco cirúrgico e anestésico ${ }^{1,2,3,6,7}$.

Dentre as técnicas por via abdominal temos: as retopexias sem sigmoidectomias (fixação sacral); retopexias com sigmoidectomias e retopexias com ou- tras técnicas. Por via perineal as seguintes técnicas podem ser utilizadas: Thiersch; Retosigmoidectomia perineal e plicatura retal com retirada do excesso da mucosa (Delorme).

A abordagem abdominal é preferível para pacientes jovens saudáveis, pois estes toleram melhor o risco anestésico cirúrgico. Devido a menor taxa de recidiva essas técnicas têm sido preferidas em detrimento das perineais ${ }^{2}$.

A abordagem perineal por sua vez é menos traumática para o paciente, porém tem um índice de recidiva maior e é indicada a pacientes com alto risco anestésico cirúrgico e uma baixa expectativa de vida.

No entanto alguns estudos têm demonstrado que a indicação da técnica de Delorme pode não ser tão restrita pelos resultados que obtiveram com sua utilização ${ }^{2}$. Em nosso paciente a correção da procidência retal pela técnica de Delorme demonstrou ser a mais adequada já que não apresentou recidiva até seis meses da cirurgia. Sendo esta técnica menos invasiva, questionamos se não seria mais vantajoso utilizá-la como primeira opção para o tratamento da procidência.

A técnica de Delorme é essencialmente uma protectomia mucosa e um procedimento de plicatura da muscular. É utilizada preferencialmente em paciente com 3 a $4 \mathrm{~cm}$ de prolapso embora a mucosa possa se estender até $15 \mathrm{~cm}$. Mesmo em pacientes idosos frágeis, a técnica de Delorme está associada a baixos índices de mortalidade. A incontinência melhora em até $69 \%$ dos pacientes. No caso relatado a técnica de sacropromontofixação, apesar de ter sido realizada de modo adequado, não se demonstrou tão eficiente como afirma a literatura , uma vez que apresentou recidiva necessitando nova cirurgia . O caso estudado está de acordo com o descrito por Fang at. al que afirma ser a técnica de Delorme comparável às técnicas convencionais em relação ao resultado pós-operatório com a vantagem de apresentar menores índices de morbimortalidade.

\footnotetext{
ABSTRACT: The rectal prolapse is a protusion of all the layers of the rectum through the anus, characterizing a clinical picture of anal tumor, initially it results of great efforts with spontaneous reduction and subsequently it results of small efforts with difficult reduction. The incidence is bigger in women. The diagnosis is essentially clinical and the treatment is surgical. Case Report: NM, 65 years, diabetic and hypertensive, subjected to sacropromontoryfixation associated to Moscowicz's technique with recurrence after one month, so the technique of Delorme was used successfully. Discussion: the abdominal procedures are indicated for patients with less surgical risk due to its bigger complexity and smaller tax of recurrence. Among the perineal proceedings perineais, the technique of Delorme has simpler execution, however with a bigger rate of recurrence. The technique of Delorme has a smaller rate of mortality and it was the best conduct for this patient.
}

Key words: Rectal prolapse, colorectal surgery, surgical procedures operative. 


\section{REFERÊNCIAS}

1. Sobrado CW; Kiss DR; Nahas SC; Araújo SEA; Seid VE; Cotti G, et al. Tratamento cirúrgico da procidencia retal: experiência e resultados tardios de 51 pacientes. Rev Hosp Clin 2004; 59(4):168-171

2. Fang CB; Candelária PAP; Klug WA; Capelhuchnik P. Resultados de tratamento do prolapso retal pela técnica de Delorme e de retopexia. Rev Assoc Med Bras 2008; 54(2):142-145

3. Santos JR. JC. Prolapso do reto aspectos clínicos e cirúrgicos. Rev bras Coloproct 2005; 25(3):272-278.

4. Santana HJ; Torres Neto JR; Vidal MAN; Santana NMBS; Oliveira Filho JJ; Santos CM; Santos SR ; SANTOS VS. Operação de Delorme com cerclagem - análise retrospectiva de 1990-1996. Rev bras Coloproct 1996; 16:129-32.

5. Nelson H; Dozois RR. Ânus. In: Beauchamp; Evers; Mattox. Sabiston: Tratado de cirurgia -as bases biológicas da prática cirúrgica moderna: Guanabara Koogan, 2003. p. 1073-1075.
6. Miranda EM . Prolapso retal In: Souza VCT . Coloproctologia .3 ed. Medsi , ano : 183-188

7. Fillmann EEP ; Fillmann HS ; Fillmann LS .Procidência do reto . In: Reis neto J A . Coloproctologia atual. HTTP:// www.proctosite.com/library/books/livro_reis_novo/ index_coloproc_atual.htm

8. Cesar MAP; Ortiz J A ; Aguida H A; Capelhuchnik P ; Fang CB ; Klug WA. A presença de retocele interfere nos exames de fisiologia anal ? Rev bras Coloproct 2008; 29(4):345-349.

\section{Endereço para correspondência:}

MARIA AUXILIADORA PROLUNGATTI CESAR

Hospital Universitário de Taubaté - Clínica Cirúrgica

Avenida Granadeiro Guimarães, 270

Taubaté -SP

CEP: 12020-130 